\title{
Reisemedizin - Häufige und seltene Erkrankungen
}

$\mathrm{H}$ äufige Krankheiten sind häufig, und seltene Krankheiten sind selten. Wer häufig seltene und selten häufige Krankheiten diagnostiziert, stellt häufig falsche und selten richtige Diagnosen." Dieser Satz eines meiner Lehrer trifft vermutlich auf kein anderes medizinisches Fach besser zu als auf die Reisemedizin. Interessanterweise ziehen vor allem die seltenen Reisekrankheiten das Interesse auch der medizinischen Öffentlichkeit oder soll man schon sagen die Sensationslust - auf sich, während die häufigen, auf Reisen erworbenen Erkrankungen leider viel zu wenig oder meist gar nicht in der Presse erscheinen.

Ein spektakulärer Fall eines importierten Gelbfiebers oder Lassa-Fiebers erreicht eine weitaus größere Aufmerksamkeit. Wir Ärzte hängen an den Lippen des die täglichen Bulletins verkündenden Arztes und diskutieren den bekannt gegebenen Erkrankungsverlauf mit Kollegen oder stellen sogar selbst mögliche Verdachtsdiagnosen. Dabei ist es selbst für den reisemedizinisch versierten Arzt nicht immer einfach, auf dem Boden der Realität zu bleiben. Dementsprechend fällt es dem allgemein tätigen Niedergelassenen und Klinikarzt noch schwerer, seine diagnostischen Überlegungen in solchen Zeiten nicht zu sehr von aktuellen Ereignissen beeinflussen zu lassen.

Trotzdem sollte man immer bedenken, dass in Deutschland auf durchschnittlich höchstens einen Fall mit hämorrhagischem Fieber pro Jahr etwa 1000 Malaria-Erkrankungen mit mehreren Todesfällen diagnostiziert werden. Trotzdem stehen gastrointestinale Infektionen an aller erster Stelle der auf Reisen erworbenen Krankheiten: Durchschnittlich jeder dritte Reisende erkrankt mehr oder weniger lang und heftig an gastrointestinalen Problemen. Und obwohl inzwischen wirksame Impfungen zur Verfügung stehen, sind die Hepatitis A und die Hepatitis B nach wie vor die wichtigsten reiseassoziierten schwer verlaufenden Infektionskrankheiten in Deutschland. Doch wir sehen keine Scharen von Reportern, die sich um diese wirklichen Probleme in der Reisemedizin kümmern.
Sicherlich ist dieses Gebiet aber ein überlegenswerter Diskussionsstoff für den Medizinjournalismus, der jedoch auch den Kliniker am Bett eines nach einer Reise erkrankten Patienten in der akuten Situation beschäftigen muss. Denn er muss in möglichst kurzer Zeit die häufige von der seltenen Krankheit unterscheiden und entsprechende diagnostische, therapeutische und gegebenenfalls auch seuchenhygienische Maßnahmen einleiten. Für diesen, am Krankenbett stehenden Kliniker ist dieses Heft konzipiert und nach dem anfangs genannten Motto haben wir den häufigen auf Reisen erworbenen Krankheiten mehr Raum gegeben, und nur ein kleiner Teil dieser klinikarzt-Ausgabe widmet sich den seltenen - aber nichts desto trotz interessanten - Reisekrankheiten.

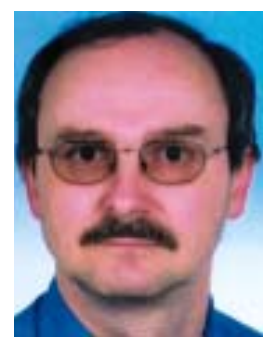

Dr. Gerhard Dobler, München (Gasteditor)

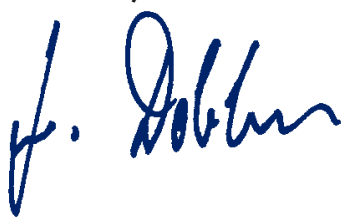

